\title{
TWO-ORBIT CONVEX POLYTOPES AND TILINGS
}

\author{
KOLYA MATTEO
}

\begin{abstract}
We classify the convex polytopes whose symmetry groups have two orbits on the flags. These exist only in two or three dimensions, and the only ones whose combinatorial automorphism group is also twoorbit are the cuboctahedron, the icosidodecahedron, and their duals. The combinatorially regular two-orbit convex polytopes are certain $2 n$ gons for each $n \geq 2$. We also classify the face-to-face tilings of Euclidean space by convex polytopes whose symmetry groups have two flag orbits. There are finitely many families, tiling one, two, or three dimensions. The only such tilings which are also combinatorially two-orbit are the trihexagonal plane tiling, the rhombille plane tiling, the tetrahedraloctahedral honeycomb, and the rhombic dodecahedral honeycomb.
\end{abstract}

\section{INTRODUCTION}

Here we will classify all convex polytopes, and face-to-face tilings of Euclidean space by convex polytopes, whose flags have two orbits under the action of the symmetry group. First we briefly define these terms.

A convex polytope is the convex hull of a finite set of points in $d$-dimensional Euclidean space $\mathbb{E}^{d}[13]$. In this paper we use " $d$-polytope" to mean " $d$ dimensional convex polytope," "polygon" to mean "2-polytope" and "polyhedron" to mean "3-polytope." A face of a convex polytope $P$ is the intersection of $P$ with a supporting hyperplane of $P$, i.e. a hyperplane $H$ such that $P$ is contained in one closed half-space determined by $H$, and such that $H$ and $P$ have non-empty intersection. We also admit the empty set and $P$ itself as "improper" faces. A face is called $j$-dimensional, or a $j$-face, if its affine hull is $j$-dimensional; the empty face is $(-1)$-dimensional. The 0 -faces are also called vertices; 1 -faces are also called edges; $(d-2)$-faces may be called ridges and $(d-1)$-faces are called facets.

The faces of $P$, ordered by containment, form a lattice $\mathcal{L}(P)$, the face lattice of $P$. The symmetry group of $P$, denoted $G(P)$, is the set of Euclidean isometries which carry $P$ to itself. The automorphism group of $P$, denoted $\Gamma(P)$, is the set of lattice isomorphisms from $\mathcal{L}(P)$ to itself. Since each transformation in $G(P)$ acts as an automorphism of $\mathcal{L}(P)$, we can consider $G(P)$ as a subgroup of $\Gamma(P)$.

Date: September 17, 2018.

2010 Mathematics Subject Classification. Primary 52B15; Secondary 51M20, 51F15, $52 \mathrm{C} 22$.

Key words and phrases. Two-orbit, convex polytopes, tilings, half-regular, quasiregular. 
A maximal chain in $\mathcal{L}(P)$ (i.e. a maximal linearly ordered set of faces) is called a flag (due to the way a vertex, followed by an edge incident to that vertex, followed by a 2 -face incident to the edge, resemble the construction of a flagpole.) The set of all flags of $P$ is $\mathcal{F}(P)$. Transformations in $G(P)$ (or automorphisms in $\Gamma(P)$ ) induce an action on $\mathcal{F}(P)$ in an obvious way. The orbits of flags under the action of $G(P)$ are called flag orbits, and a polytope with $n$ distinct flag orbits is called an $n$-orbit polytope. Similarly, orbits of flags under the action of $\Gamma(P)$ are called combinatorial flag orbits, and a polytope with $n$ such orbits is called combinatorially $n$-orbit; in the context of abstract polytopes, this is the only definition possible and the adjectives may be dropped.

In [4, p. 273], Conway et al. introduce the term flag rank for the number of flag orbits. A $k$-orbit polytope is said to have a flag rank of $k$, and they also suggest that such a polytope be called $\frac{1}{k}$-regular. Thus, in this paper we determine all the half-regular convex polytopes.

One-orbit polytopes are the regular polytopes. It is well known [8] that there are infinitely many regular polygons, namely the regular $n$-gon for each $n \geq 3$; there are five regular polyhedra, the Platonic solids; there are six regular 4-polytopes; and there are three regular $d$-polytopes for all $d>4$.

As far as flags are concerned, two-orbit polytopes are as close to regular as possible while not being regular. Two-orbit convex polytopes can either be combinatorially two-orbit, if $G(P)=\Gamma(P)$, or combinatorially regular, in which case $G(P)$ is a subgroup of index 2 in $\Gamma(P)$. In the more general case of abstract polytopes, combinatorially two-orbit polyhedra were examined by Hubard [18]. The chiral polytopes are notable examples of two-orbit abstract polytopes [26]. However, convex polytopes cannot be chiral [26, p. 496].
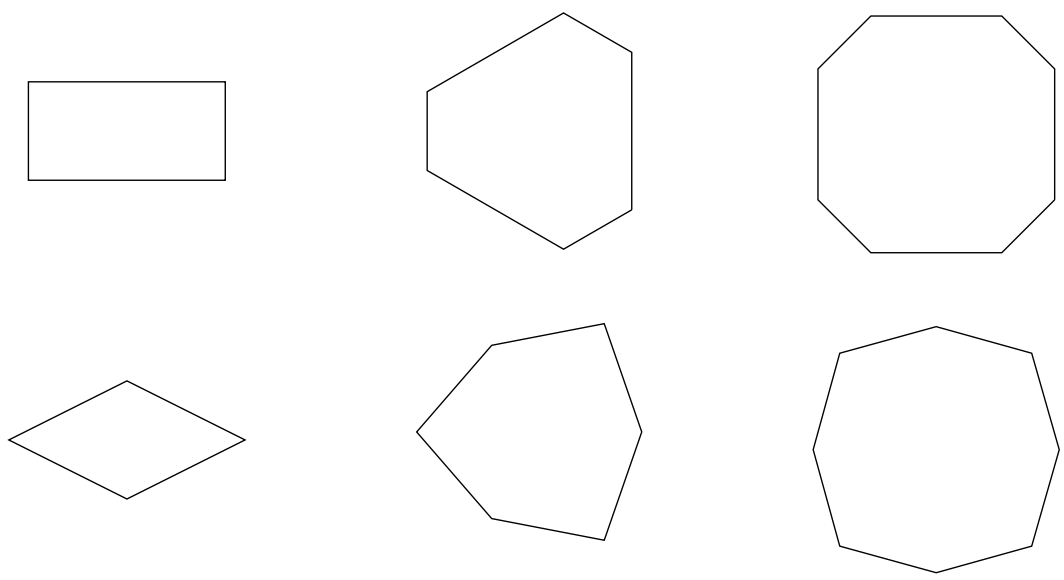

Figure 1. The first few two-orbit convex polygons, in pairs of duals

As we shall show, two-orbit convex polytopes turn out to be even scarcer than one-orbit convex polytopes, and exist only in two or three dimensions. 
There are infinitely many in two dimensions. For each $n \geq 2$, a two-orbit $2 n$ gon may be constructed by alternating edges of two distinct lengths, with the same angle at each vertex (namely the interior angle of a regular $2 n$ gon, $\frac{n-2}{n} \pi$ ). Dual to each of these is another type of two-orbit $2 n$-gon, with uniform edge lengths, but alternating angle measures.

In three dimensions, there are just four: The cuboctahedron, its dual the rhombic dodecahedron, the icosidodecahedron, and its dual the rhombic triacontahedron. We summarize the results in Theorems 1 and 2 ,

Theorem 1. There are no two-orbit d-polytopes if $d \geq 4$ (or if $d \leq 1$ ). There are exactly four, if $d=3$ : the cuboctahedron, icosidodecahedron, rhombic dodecahedron, and rhombic triacontahedron. If $d=2$, there are two infinite series of $2 n$-gons, for each $n \geq 2$. Polygons of one series alternate between two distinct edge lengths. Polygons of the other alternate between two distinct angle measures.

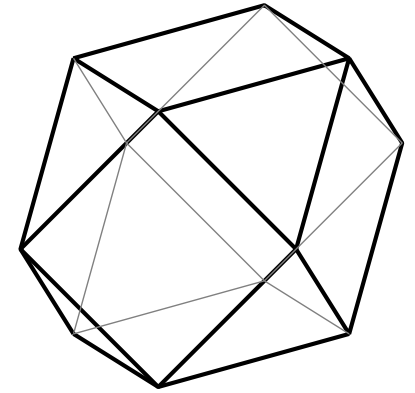

Cuboctahedron

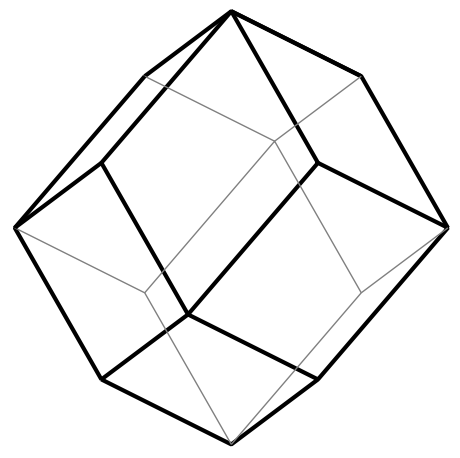

Rhombic Dodecahedron

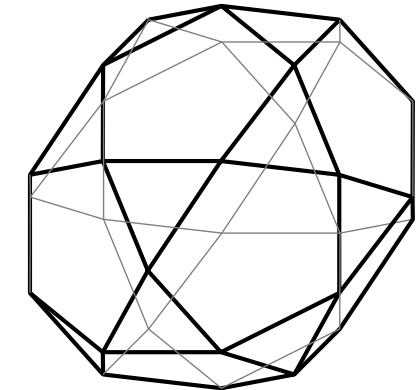

Icosidodecahedron

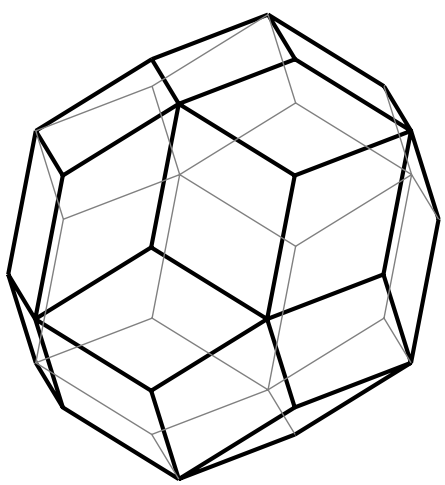

Rhombic Triacontahedron

Figure 2. The two-orbit convex polyhedra

In Section 6 we classify all two-orbit tilings by convex polytopes. We consider only face-to-face, locally finite tilings. See Section 6 for a description of each of the named tilings. 
Theorem 2. There are no two-orbit tilings of $\mathbb{E}^{d}$ if $d \geq 4$ (or if $d=0$ ). If $d=1$, there is one family: an apeirogon alternating between two distinct edge lengths. If $d=2$, there are four: the trihexagonal tiling (3.6.3.6); its

dual, the rhombille tiling; a family of tilings by translations of a rhombus; and a family of tilings by rectangles. If $d=3$, there are two: the tetrahedraloctahedral honeycomb and its dual, the rhombic dodecahedral honeycomb.

In the above two theorems, all those examples which vary by a real parameter greater than one (both types of $2 n$-gons, the apeirogon, and the tilings by rhombi and rectangles) are combinatorially regular; in each case, allowing the parameter to become one yields a regular polygon or tiling, to which all other members of the family are isomorphic. The other examples, namely the four polyhedra, the trihexagonal tiling, the rhombille tiling, the tetrahedral-octahedral honeycomb, and the rhombic dodecahedral honeycomb, are all unique (up to similarity), and are all combinatorially two-orbit.

\section{Preliminary Facts}

Let $P$ be a $d$-polytope. Recall that flags are maximal chains of faces of $P$. Two flags are said to be adjacent if they differ in exactly one face; if they differ in the $j$-face, they are said to be $j$-adjacent. The face lattice $\mathcal{L}(P)$ satisfies the following four properties, which are in fact taken to be the definition of an abstract polytope of rank $d$ [22, p. 22]:

(P1) There is a least face $F_{-1}$, the empty face, and a greatest face $F_{d}$, which is $P$ itself.

(P2) Every flag contains $d+2$ faces.

(P3) (Strong flag-connectivity:) For any two flags $\Phi$ and $\Psi$ of $P$, there exists a sequence of flags $\Phi=: \Phi_{0}, \Phi_{1}, \ldots, \Phi_{k}:=\Psi$, such that each flag is adjacent to its neighbors and $\Phi \cap \Psi \subseteq \Phi_{i}$ for each $i$.

(P4) (The diamond condition:) For any $j, 1 \leq j \leq d$, any $j$-face $G$ of $P$, and any $(j-2)$-face $F$ contained in $G$, there are exactly two faces $H$ such that $F<H<G$.

A $d$-polytope $Q$ is said to be dual to $P$ if the face lattice $\mathcal{L}(Q)$ is antiisomorphic to the lattice $\mathcal{L}(P)$, that is, identical to $\mathcal{L}(P)$ with the order reversed. A bijective, order-reversing function $h: \mathcal{L}(P) \rightarrow \mathcal{L}(Q)$ is called a duality. A dual polytope to $P$ is often denoted $P^{*}$. Clearly, any two duals of $P$ are combinatorially isomorphic. A dual $P^{*}$ to any convex polytope $P$ may be constructed by the process of polar reciprocation: After translating $P$, if necessary, so that the origin is contained in its interior, let $P^{*}=\bigcap_{y \in P}\{x$ $\mid\langle x, y\rangle \leq 1\}$, where $\langle x, y\rangle$ is the scalar product. Then $\left(P^{*}\right)^{*}=P$ and $G\left(P^{*}\right)=G(P)$. Thus, when necessary, we may assume that a polytope and its dual have the same symmetry group.

For any two faces $F$ and $G$ of $P$ with $F \leq G, G / F$ denotes the section of $\mathcal{L}(P)$ whose face lattice is $\{H \in \mathcal{L}(P) \mid F \leq H \leq G\}$. This section may be realized as a convex polytope by taking the dual polytope $G^{*}$ to $G$, say 
with a duality $h: G \rightarrow G^{*}$; then the dual $h(F)^{*}$ to the face $h(F)$ of $G^{*}$ is the desired polytope.

A subgroup of $G(P)$ acts on the section $G / F$; namely, those symmetries which fix all faces of $P$ which contain $G$ and all faces of $P$ which are faces of $F$. These form a subgroup which acts faithfully on $G / F$ in a well-defined way. As symmetries of $G / F$, this group is a subgroup of the symmetry group of $G / F$. We call it the restricted subgroup, denoted $G_{P}(G / F)$ (this is not standard notation.)

Note that the symmetry group of a two-orbit $d$-polytope $P$ can have at most two orbits on its $j$-faces, for any $j<d$.

Claim 1. Suppose $P$ is a two-orbit d-polytope. If the symmetry group $G(P)$ is not transitive on $j$-faces for some $j$, then $G(P)$ is transitive on $i$-faces for all $i \neq j$, where $0 \leq i, j \leq d-1$.

Proof. Otherwise, we have two orbit classes of $i$-faces, say class I and II, and two classes of $j$-faces, say A and B. Without loss of generality, suppose $j<i$. Let us say that a flag of $P$ whose $j$-face is in class A and whose $i$-face is in class I is an A-I flag, and similarly for other cases. Then we have more than two flag types, A-I, A-II, B-I, and B-II, unless the $j$-faces in class A occur only in one class of $i$-faces, say $\mathrm{I}$, and $j$-faces in class B occur only in $i$-faces in class II. But, as we will show, this violates the connectivity property (P3).

Let $\Phi$ be an A-I flag and $\Psi$ be a B-II flag. By flag-connectedness there is a sequence of adjacent flags, $\Phi=\Phi_{0}, \Phi_{1}, \ldots, \Phi_{k}=\Psi$. Let $\ell$ be the least index such that $\Phi_{\ell}$ contains a $j$-face in class B or an $i$-face in class II, or both. Then $\Phi_{\ell-1}$ is an A-I flag, and since $\Phi_{\ell}$ is adjacent to $\Phi_{\ell-1}$, only one face is different, so $\Phi_{\ell}$ is either an A-II flag or a B-I flag. Therefore $P$ has at least three flag orbits.

Polytopes which are transitive on $j$-faces for all $1 \leq j \leq d-1$ are called fully transitive. It is a theorem of McMullen's thesis [21] that fully transitive convex polytopes are regular.

Theorem 3 (McMullen [21, 4C6]). A d-polytope $P$ is regular if and only if for each $j=0, \ldots, d-1$, its symmetry group $G(P)$ is transitive on the $j$-faces of $P$.

Therefore, for a two-orbit $d$-polytope $P$ there is a $j, 0 \leq j \leq d-1$, so that $G(P)$ is not transitive on the $j$-faces but is transitive on the faces of every other rank. We shall call such a polytope $j$-intransitive.

In the language of Hubard [18], a 0-intransitive two-orbit polyhedron is of class $2_{1,2}$, a 1 -intransitive two-orbit polyhedron is of class $2_{0,2}$, and a 2 intransitive two-orbit polyhedron is of class $2_{0,1}$. Claim 1 and the above comments were proved in [18]. They are consequences of Theorem 5 therein, which we may paraphrase to say that an (abstract) two-orbit $d$-polytope $P$ is either fully transitive, or there exists a $j(1 \leq j \leq d)$ such that $P$ is $i$-transitive for every $i \neq j$, but not for $i=j$. In using any results about abstract two-orbit polytopes, however, we must be careful to remember that 
convex two-orbit polytopes may be combinatorially regular and not combinatorially two-orbit.

Claim 2. For any convex polytope $P$, the order of the symmetry group $G(P)$ divides the number of flags of $P$. Each flag orbit has the same size, namely $|G(P)|$, and so $P$ is a two-orbit polytope if and only if the number of flags is twice the order of $G(P)$.

Proof. This all follows from the fact that $G(P)$ acts freely on the set of flags of $P$. Let $\Phi$ be any flag of $P$. Any $\gamma \in G(P)$ acts on the $j$-adjacent flag $\Phi^{j}$ to $\Phi$ as $\gamma\left(\Phi^{j}\right)=\gamma(\Phi)^{j}$, since $\gamma$ is an automorphism of the face lattice. Therefore, if $\gamma \in G(P)$ is such that $\gamma(\Phi)=\Phi$, then $\gamma$ will also fix each flag adjacent to $\Phi$, and thus all flags of $P$ by flag-connectedness, so $\gamma$ is the identity.

It follows that the dual to a two-orbit polytope is two-orbit; the dual to a $j$-intransitive $d$-polytope is $(d-j-1)$-intransitive.

Claim 3. If $P$ is a two-orbit $j$-intransitive d-polytope, and $\Phi$ is any flag, then for any $i \neq j$ the $i$-adjacent flag $\Phi^{i}$ is in the same orbit as $\Phi$. That is, there exists a symmetry $\rho \in G(P)$ such that $\rho(\Phi)=\Phi^{i}$.

Proof. Since there are only two flag orbits, and two classes of $j$-faces, the orbit of a given flag is determined entirely by its $j$-face. For $i \neq j, \Phi$ and $\Phi^{i}$ share their $j$-face, hence are in the same flag orbit.

Corollary 1. If $P$ is a two-orbit $j$-intransitive d-polytope, and $\Phi$ is any flag, then the $j$-adjacent flag $\Phi^{j}$ is not in the same flag orbit as $\Phi$.

Proof. If $\Phi^{j}$ were in the same orbit as $\Phi$, then by Claim [3, for each $i=$ $0, \ldots, d-1$ there exists an isometry $\rho_{i}$ of $P$ such that $\rho_{i}(\Phi)=\Phi^{i}$. But if a flag is in the same orbit as all of its adjacent flags, it follows from flagconnectedness that $P$ is regular (see Proposition 2B4 of [22] or Theorem 4B1 of [21].)

The next corollary is immediate from Corollary 1 .

Corollary 2. If $P$ is a two-orbit $j$-intransitive $d$-polytope, then for any $(j+1)$-face $F_{j+1}$ of $P$ and any $(j-1)$-face $F_{j-1}$ contained in $F_{j+1}$, the two $j$-faces $H$ with $F_{j-1}<H<F_{j+1}$ are in different $j$-face orbits.

In the following, by "chain of cotype $\{j\}$ " we mean a chain of faces in $\mathcal{L}(P)$ including a face of each rank except $j$.

Claim 4. If $P$ is a two-orbit $j$-intransitive d-polytope, then $G(P)$ acts transitively on chains of cotype $\{j\}$.

Proof. Let $\Psi$ and $\Omega$ be two chains of cotype $\{j\}$. By Corollary 2 , the two $j$-faces which are incident to the $(j-1)$-face and $(j+1)$-face of $\Psi$ are in different $j$-face orbits. Recall that the orbit of a given flag is determined entirely by its $j$-face. So we may extend $\Psi$ to a flag in either flag orbit. 
Similarly, we may extend $\Omega$ to a flag in either orbit. Thus, we extend $\Psi$ to a flag $\Psi^{\prime}$ and $\Omega$ to a flag $\Omega^{\prime}$ such that both are in the same orbit; then there is a symmetry $\gamma \in G(P)$ so $\gamma\left(\Psi^{\prime}\right)=\Omega^{\prime}$, and thus $\gamma(\Psi)=\Omega$.

Claim 5. If $P$ is a two-orbit $j$-intransitive d-polytope, then $j=0$ or $j=$ $d-1$.

Proof. Suppose $1 \leq j \leq d-2$. Then there is a $(j-2)$-face $F_{j-2}$ contained in some $(j+2)$-face $F_{j+2}$ in $P$. The section $Q=F_{j+2} / F_{j-2}$ is a polyhedron. By Claim 4, isometries in the restricted group $G_{P}(Q)$ act transitively on the vertices and facets of $Q$ (corresponding to $(j-1)$-faces and $(j+1)$-faces of $P$, respectively.) By vertex transitivity, every vertex is in the same number $q$ of edges. By Corollary 2, the edge orbits alternate across each facet, so $q$ is even. By facet transitivity, each facet is a $p$-gon for some $p$, and again by Corollary 2 the edge orbits alternate at each vertex, so $p$ is even.

However, this contradicts Euler's theorem. In fact, each polyhedron without triangular facets has at least one 3 -valent vertex [13, p. 237].

Claim 6. If $P$ is a two-orbit $j$-intransitive $d$-polytope, then all $i$-faces, for $i \leq j$, are regular. More generally, any section $G / F$, where $G$ is a $k$-face and $F$ is an $l$-face, is regular if $j \leq l$ or $k \leq j$. If $l<j<k$, then $G / F$ has two flag orbits under the restricted subgroup $G_{P}(G / F)$.

Proof. Since there are only two flag orbits, and two classes of $j$-faces, the orbit of a given flag is determined entirely by its $j$-face. Suppose $G / F$ is a section as described and we do not have $l<j<k$. Choose a base flag $\Phi$ of $G / F$ and extend it to a flag $\Phi^{\prime}$ of $P$. Now any flag $\Psi$ of $G / F$ may be extended to a flag $\Psi^{\prime}$ of $P$ which agrees with $\Phi^{\prime}$ for all $i$-faces with $i \leq l$ or $i \geq k$. In particular, $\Phi^{\prime}$ and $\Psi^{\prime}$ share the same $j$-face, so there is an isometry $\gamma \in G(P)$ such that $\gamma\left(\Phi^{\prime}\right)=\Psi^{\prime}$. Then $\gamma$ restricts to a symmetry of $G / F$ carrying $\Phi$ to $\Psi$. Hence $G / F$ is regular.

On the other hand, if $l<j<k$, then $G / F$ contains a $(j-1)$-face $F_{j-1}$ of $P$ and a $(j+1)$-face $F_{j+1}$ of $P$ which contains $F_{j-1}$. By Corollary 2 , the two $j$-faces $H$ of $P$ with $F_{j-1}<H<F_{j+1}$ are in different orbits. Thus $G / F$ has at least two flag orbits under those isometries in $G(P)$ which restrict to $G / F$. On the other hand, for any two flags $\Phi$ and $\Psi$ of $G / F$ which contain the same kind of $j$-face of $P$, we may extend these to flags $\Phi^{\prime}$ and $\Psi^{\prime}$ of $P$ which agree on all $i$-faces with $i \leq l$ and $i \geq k$. Then an isometry $\gamma \in G(P)$ exists with $\gamma\left(\Phi^{\prime}\right)=\left(\Psi^{\prime}\right)$, and this $\gamma$ restricts to $G / F$ where it takes $\Phi$ to $\Psi$. Hence $G / F$ has two flag orbits under those transformations in $G(P)$ which restrict to $G / F$.

Note that those sections in Claim [6] with two flag orbits under the restricted subgroup are either two-orbit polytopes or regular. Their full group of symmetries includes the restricted subgroup, but may be bigger. If the section is in fact two-orbit, then its symmetry group agrees with the restricted subgroup. In particular, if a face $F$ of a two-orbit $j$-intransitive 
polytope is two-orbit, then $F$ is also $j$-intransitive; note than then $j=0$, by Claim 5 .

\section{Two Dimensions}

Suppose $P$ is a two-orbit polygon. If $P$ does not have all edges of the same length, then it has two distinct edge lengths; if it had three or more, then there would be three or more flag orbits. In this case, $P$ is not edgetransitive, so it must be vertex-transitive. Then no two edges of the same length may be adjacent, since in that case, by vertex-transitivity, all edges would be the same length. So $P$ must alternate edges of two distinct lengths, and by vertex-transitivity all angles are the same.

On the other hand, suppose $P$ does have all edges the same length. If the angle at each vertex is the same, then $P$ would be regular. Therefore, $P$ has at least two distinct angles; it has at most two, since there at most two vertex orbits. Then $P$ is not vertex-transitive, so it must be edge-transitive, which implies that $P$ alternates between two distinct angles.

We have shown that every two-orbit convex polygon must be of one of the two types described above. It is not hard to see that, moreover, such $2 n$-gons exist for each $n \geq 2$. The existence of non-regular rectangles is well known. For each $n \geq 3$, a polygon of the first type may be constructed from a regular $n$-gon by truncation, i.e. chopping off a corner at each vertex. In the top row of Figure 1, you may see how the hexagon is a truncated equilateral triangle, and the octagon is a truncated square.

The existence of each $2 n$-gon of the second type is then clear, since they are the duals of the polygons of the first type; i.e. they may be constructed by taking the convex hull of vertices placed at the midpoint of each edge of a polygon of the first type.

It is also clear that such polygons are, indeed, two-orbit. Let us consider a polygon $P$ of the first type. It then follows for the second type by duality. Since $P$ is not edge-transitive, it has at least two flag orbits. Since $P$ is a truncated regular $n$-gon, it has (at least) all the symmetries of the regular $n$-gon, which has order $2 n$. But $P$ has $4 n$ flags ( $2 n$ vertices, each in 2 edges), so $P$ has at most $4 n / 2 n=2$ flag orbits. Therefore $P$ is a two-orbit polygon.

\section{Three Dimensions}

A quasiregular polyhedron is vertex-transitive and has exactly two kinds of facets, which are regular and alternate around each vertex. By Claims 1 and 6. any 2-intransitive two-orbit polyhedron is vertex-transitive, edgetransitive, and has regular facets in two orbits. The two types of facet must alternate around each vertex, i.e. each edge must be incident to one facet of each type, by edge-transitivity. Thus any 2-intransitive two-orbit polyhedron is quasiregular. But there are only two quasiregular polyhedra: the cuboctahedron and the icosidodecahedron, two of the Archimedean solids [8, p. 18]. 
We may verify that these are two-orbit polyhedra. The cuboctahedron has at least two flag orbits, since it is not regular, having both square and triangular faces. It has 12 vertices, each incident to 4 edges, and each edge is in 2 faces, so it has $12 \cdot 4 \cdot 2=96$ flags. The cuboctahedron may be formed by truncating each vertex of the 3-cube at the midpoints of the edges, so it retains all the symmetries of the cube, a group of order 48 . Hence the cuboctahedron has at most $96 / 48=2$ orbits, and thus is a twoorbit polyhedron (and also combinatorially two-orbit.)

The icosidodecahedron has at least two flag orbits, since it is not regular, having both triangular and pentagonal faces. It has 30 vertices, each in 4 edges, and each edge is in 2 faces, so it has $30 \cdot 4 \cdot 2=240$ flags. The icosidodecahedron may be formed by truncating each vertex of the dodecahedron at the midpoints of the edges, so it retains all the symmetries of the dodecahedron, a group of order 120. Hence the icosidodecahedron has at most $240 / 120=2$ orbits, and thus is a two-orbit polyhedron (and also combinatorially two-orbit.)

Any two-orbit polyhedron which is 0-intransitive must be dual to one of these two, so we have the rhombic dodecahedron, dual to the cuboctahedron, and the rhombic triacontahedron, dual to the icosidodecahedron. As duals to Archimedean solids, these are Catalan solids.

Rather than using the list of quasiregular polyhedra, it is possible to arrive at candidates for 0 -intransitive or 2-intransitive two-orbit polyhedra by considering all the edge-transitive polyhedra. It turns out there are only nine: the five platonic solids, the cuboctahedron, the icosidodecahedron, the rhombic dodecahedron, and the rhombic triacontahedron [12; 15].

By Claim [5, there are no 1-intransitive two-orbit polyhedra. In fact, polyhedra which are vertex-transitive and facet-transitive have a name, the noble polyhedra, and the only non-regular ones (i.e. the 1-intransitive polyhedra) are disphenoid tetrahedra, which are tetrahedra with non-equilateral triangular faces [3, p. 26]. It is not hard to see that, if not regular, a tetrahedron has at least three flag orbits.

Hence the cuboctahedron, icosidodecahedron, rhombic dodecahedron, and rhombic triacontahedron are the only two-orbit polyhedra. The same result is found in Orbanić, Pellicer, and Weiss [24, p. 427] as a consequence of Theorem 6.1 therein, stating that every 2-orbit map on the sphere is either the medial of a regular map on the sphere, or dual to one.

\section{Higher Dimensions}

Suppose $P$ is a $j$-intransitive two-orbit $d$-polytope with $d \geq 4$; by Claim 5 $j$ is either 0 or $d-1$. Any two-orbit 0 -intransitive polytope is dual to a twoorbit $(d-1)$-intransitive polytope, so we shall restrict our attention to the latter case. Such a polytope is vertex-transitive, and by Claim 6 has regular facets. This is the definition used by Gosset [11] for semiregular polytopes. 
In his 1900 paper he gives a complete list of all the semiregular polytopes. The list was proved to be complete in Blind and Blind [1].

There are only seven semiregular convex polytopes in dimensions greater than three. There are three 4-polytopes: the rectified 4-simplex, the snub 24-cell, and the rectified 600-cell. The rectified 4-simplex, which Gosset called "tetroctahedric," is the convex hull of the midpoints of the edges of the 4-simplex. The facets are tetrahedra and octahedra. It has 360 flags, with 10 vertices, each in 6 edges, each edge in 3 ridges, and each ridge in 2 facets. It has the same symmetry group as the 4 -simplex, of order 120; hence it has three flag orbits.

The rectified 600-cell, which Gosset called "octicosahedric," is the convex hull of the midpoints of the edges of the 600-cell. The facets are octahedra and icosahedra. It has 43,200 flags, with 720 vertices, each in 10 edges, each edge in 3 ridges and each ridge in 2 facets. It has the same symmetry group as the 600-cell, of order 14,400; hence it has three flag orbits.

The snub 24-cell, which Gosset called "tetricosahedric," has icosahedra and tetrahedra for facets. It has 96 vertices, each in 9 edges; 6 of these edges are in 3 ridges, and the other 3 edges are in 4 ridges. (This already makes it clear that there are at least two orbit classes of edges, as well as at least two orbit classes of facets, so it cannot be two-orbit.) Each ridge is in 2 facets. Hence there are 5,760 flags. It has half the symmetries of the 24-cell, leaving 576. So it has ten flag orbits.

The remaining examples form Coxeter's $k_{21}$ family [8, $\S 11.8$; 7], with one each in dimensions 5 through 8 . They are the 5 -demicube, or $1_{21}$, Gosset's "5-ic Semi-regular"; $2_{21}$ or "6-ic Semi-regular"; $3_{21}$ or "7-ic Semi-regular"; and $4_{21}$ or "8-ic Semi-regular". Each of these has the preceding one for its vertex figure, starting with the rectified 4-simplex (which may also be called $0_{21}$ ) as the vertex figure of the 5 -demicube. Of course, by Claim 6 , if any member of this family were two-orbit, then the previous member (being a section) would either be two-orbit or regular. So by induction, none of these polytopes are two-orbit. In fact, each has three flag orbits.

Thus, no two-orbit convex polytopes exist in more than three dimensions.

In [4, pp. 409-411], Conway et al. say that the $n$-dimensional demicube, i.e. the convex hull of alternate vertices of the $n$-cube (which they call a hemicube), has $n-2$ flag orbits. So the 4-demicube should be two-orbit. The 4-demicube is described specifically as a 4-crosspolytope "but with only half its symmetry." This apparently contradicts our result!

However, if the 4 -cube has for its vertices the 16 points in $\mathbb{E}^{4}$ with all coordinates 0 or 1 , then the vertices of the 4 -demicube are $(0,0,0,0),(1,1,1,1)$, and all vectors with two 0's and two 1's. Hence if $x$ is a vertex, so is $\mathbf{1}-x$, where $\mathbf{1}=(1,1,1,1)$. Grouping the 8 vertices in pairs $(x, \mathbf{1}-x)$, we find four axes which are mutually perpendicular. Thus we have four antipodal pairs of vertices of a regular 4-crosspolytope. Hence the "two-orbit" 4-demicube is actually a regular 4-crosspolytope with artificially restricted symmetries, 
essentially by coloring the facets depending whether they were formed inside a facet, or at a missing vertex, of the 4-cube.

\section{TILINGS}

A tiling of $d$-dimensional Euclidean space $\mathbb{E}^{d}$, also called a tessellation or a honeycomb, is a countable collection of subsets (called tiles) of $\mathbb{E}^{d}$ which cover $\mathbb{E}^{d}$ without gaps or overlaps; that is, the union of the tiles is $\mathbb{E}^{d}$, and the interiors of the tiles are pairwise disjoint. Here, we consider only locally finite face-to-face tilings by convex polytopes, meaning that all the tiles must be convex polytopes, every compact subset of $\mathbb{E}^{d}$ meets only finitely many tiles, and the intersection of any two tiles is a face of both (possibly the empty face). The face lattice of a tiling of $d$-dimensional space meets all the criteria defining an abstract polytope of rank $d+1$, and we call it a rank $(d+1)$ tiling. The $d$-dimensional tiles are the facets. A rank 3 tiling is called a plane tiling, and a rank 2 tiling is called an apeirogon. The latter necessarily consists of infinitely many edges (line segments) covering the line, and has been described as the limit of a sequence of $n$-gons as $n \rightarrow \infty$.

A normal tiling has

- tiles which are homeomorphic to closed balls,

- two positive radii $r$ and $R$ such that every tile contains a ball of radius $r$ and is contained in a ball of radius $R$, and

- the property that the intersection of any two tiles is empty or connected.

A two-orbit tiling has at most two congruence classes of tiles, so that the tiles are uniformly bounded (above and below) by balls of two given radii; together with convex polytopes as tiles, this is sufficient to establish that the tiling is normal. This rules out certain pathological possibilities for tilings.

Claim 1 still applies: if a two-orbit tiling is not fully transitive, then it is not transitive on the faces of exactly one dimension, say $j$, and we call it $j$-intransitive. However, Theorem 3 does not apply; the proof depends on the fact that the vertices of a vertex-transitive polytope lie on a sphere, which is not the case for a tiling. So fully transitive two-orbit tilings are a possibility (and some exist.) Claim 2 no longer makes sense, since the symmetry group and the set of flags are both infinite, but Claim 3 and its corollaries still hold for any $j$-intransitive two-orbit tilings. Finally, Claim 6 applies: the faces and sections of a two-orbit tiling have at most two orbits.

Following [16], we say two tilings are equal if one can be mapped onto the other by a uniform scale transformation followed by an isometry.

6.1. Apeirogons. There is one two-orbit tiling of the line, which varies by a single real parameter greater than one: an apeirogon alternating between two distinct edge lengths. Note that the construction of well-behaved duals does not work, in general, for tilings, as it does for polytopes. For example, if one constructs a "dual" to this two-orbit apeirogon by taking edge midpoints for vertices, one obtains a regular apeirogon, which is then self-dual! 
This tiling is combinatorially regular.

6.2. Plane tilings. We consider four cases of plane tilings, based on their transitivity properties.

6.2.1. Fully transitive. Grünbaum and Shephard [16] contains the full list of isohedral (i.e. tile-transitive) plane tilings (Table 6.1), isotoxal (i.e. edgetransitive) plane tilings (Table 6.4), and isogonal (vertex-transitive) plane tilings (Table 6.3). There are only four plane tilings realizable by convex tiles which have all three properties: the three regular plane tilings and a tiling by translations of a rhombus, labeled IH74 as an isohedral tiling, IG74 as an isogonal tiling, and IT20 as an isotoxal tiling. On [16, p. 311] it is confirmed that this rhombus tiling is the only non-regular fully transitive tiling realizable by convex tiles. Figure 3 shows a portion of this tiling, with flags of one orbit shaded. For a given flag $\Phi$, both the 0 -adjacent flag $\Phi^{0}$ and the 2-adjacent flag $\Phi^{2}$ are in the other orbit, whereas the 1-adjacent flag $\Phi^{1}$ remains in the same orbit; thus with the notation of Hubard [18] this tiling is in class 2 .

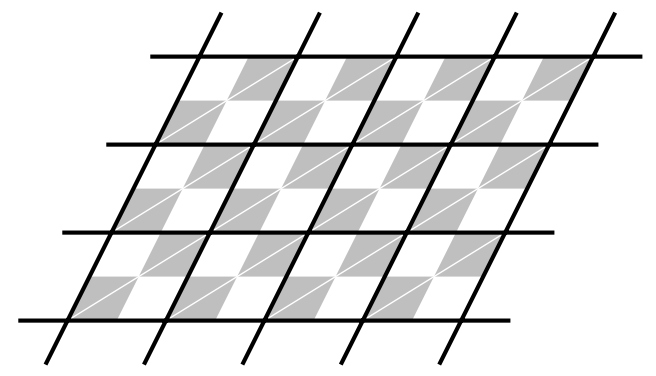

FiguRE 3. The fully-transitive rhombus tiling

A family of unequal versions of this tiling may be obtained by varying a single real parameter greater than one (the ratio of the diagonals of the rhombus.) The tiling is self-dual when taking tile midpoints for vertices. It is combinatorially regular.

6.2.2. 2-intransitive. The facets of a 2-intransitive two-orbit tiling must be regular, by Claim 6. By edge-transitivity, the two facets bordering each edge are from different orbits; hence they alternate around each vertex. By vertex-transitivity, each vertex appears in the same kinds of tiles, which appear in the same order around each vertex; a common notation for such a situation is (p.q.r...) to indicate that each vertex $v$ is in a $p$-gon adjacent to a $q$-gon (containing $v$ ) adjacent to an $r$-gon, etc. An exponent may be used to indicate repetition; for instance, the regular tiling by equilateral triangles, (3.3.3.3.3.3), is denoted $\left(3^{6}\right)$.

If six facets appear at each vertex, then they must all be triangles, since replacing any triangle by a regular $n$-gon with $n \geq 4$ will not fit in the plane. 
The only tiling with six equilateral triangles at every vertex is the regular tiling $\left(3^{6}\right)$. Hence there must be exactly four facets at each vertex.

If none of the facets are triangles, then each has at least four sides. Four squares fit exactly around a vertex, but replacing any squares by regular $n$-gons with $n \geq 5$ will not fit in the plane. The only tiling with four squares at every vertex is the regular tiling $\left(4^{4}\right)$. Hence there must be at least some triangles.

If all four faces at each vertex are equilateral triangles, there is too much angular deficiency to tile the plane; indeed, the only such figure is the regular octahedron, $\left(3^{4}\right)$.

If triangles alternate with squares, the resulting figure is the cuboctahedron, (3.4.3.4). If triangles alternate with pentagons, the resulting figure is the icosidodecahedron, (3.5.3.5). (This is, in brief, the proof that these are the only quasiregular polyhedra.)

If triangles alternate with hexagons, we do obtain a plane tiling, denoted (3.6.3.6). This is one of the 11 uniform plane tilings, also called Archimedean tilings. This tiling, seen in Figure 4, is sometimes called "trihexagonal" or "hexadeltille."

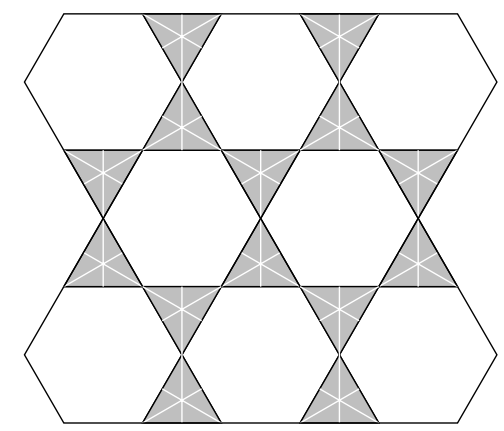

FiguRE 4. The trihexagonal tiling

If we replace the hexagons by regular $n$-gons with $n \geq 7$, the total angles are excessive to fit in the plane. Hence (3.6.3.6) is the unique two-orbit 2-intransitive plane tiling. Coxeter [8, p. 60] calls it by the extended Schläfli symbol $\left\{\begin{array}{l}3 \\ 6\end{array}\right\}$, which is suggestive of the construction by taking the midpoints of the edges of the regular tiling $\{3,6\}$, or equivalently of its dual, the regular tiling $\{6,3\}$. He describes it as a quasiregular tessellation.

It is combinatorially two-orbit. Taking the dual by using tile midpoints for vertices works well and results in the rhombille tiling detailed below.

6.2.3. 1-intransitive. By facet-transitivity, each facet has the same number of sides, say $p$, and by vertex-transitivity, each vertex is incident to the same number of edges, say $q$. Thus a 1-intransitive plane tiling has a Schläfli symbol $\{p, q\}$. Since edges of the two orbits alternate at each vertex of a tile, $p$ and $q$ are both even; the only possible symbol is $\{4,4\}$. The tiles 
must be regular or two-orbit. The only tiling by squares is regular; so the tiles must be two-orbit 4-gons, i.e. rectangles or rhombi.

It follows from vertex-transitivity, or from adding angle defects, that rhombi must be arranged with two acute angles and two obtuse angles at each vertex. In the case that the two angle types alternate, we obtain the tiling in Figure 3, which we know to be fully transitive. In the case that the obtuse angles are adjacent to each other, and the acute angles are adjacent to each other, we do obtain a 1-intransitive plane tiling. The rhombi are arranged in strips which alternate direction. However, this tiling actually has four orbits. Indeed, in a 1-intransitive two-orbit tiling, the orbit of a flag is determined entirely by the edge it contains; if any face is also two-orbit, so that its symmetry group is the same as the restricted subgroup, then its flag orbits must also be determined by edges, and not vertices as in the case of a rhombus.

This leaves only the tiling by copies of a rectangle. This is the unique two-orbit 1-intransitive family of plane tilings, and varies by a single real parameter greater than one. It is self-dual and combinatorially regular, being isomorphic to the square tiling $\left(4^{4}\right)$. Figure 5 shows a patch of this tiling, with flags of one orbit shaded.

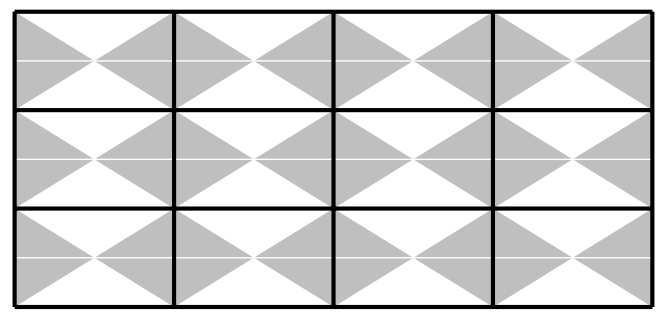

FIGURE 5. The 1-intransitive rectangle tiling

6.2.4. 0-intransitive. It is tempting to say that any 0-intransitive tiling must be dual to a 2-intransitive one. However, Grünbaum and Shephard [16] admonish us that for tilings, no duality theorem exists which would allow us to make such statements! Nonetheless, it turns out that the only 0intransitive two-orbit tiling is indeed dual to the uniform tiling (3.6.3.6). We can confirm this by again turning to the tables of isohedral and isotoxal tilings in [16]; the only additional tiling realizable by convex tiles with both properties is denoted IH37 as an isohedral tiling and IT11 as an isotoxal tiling.

This is a tiling by copies of a rhombus, which can be viewed as dividing the hexagons of the regular tiling $\left(6^{3}\right)$ into three rhombi each. It is called "rhombille" or "tumbling blocks," and is familiar as the visual illusion of a stair-case of blocks which can be seen in two ways. It is combinatorially two-orbit. 


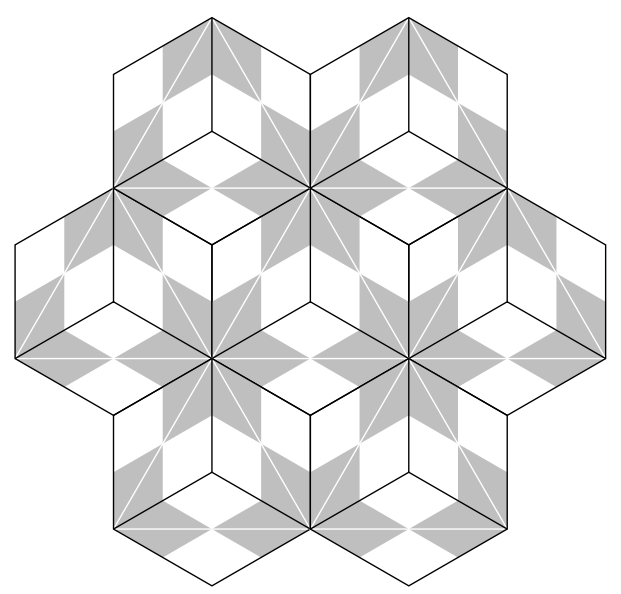

FIGURE 6 . The rhombille tiling

6.3. Tilings of three-space. A tiling in $\mathbb{E}^{d}$ is said to be uniform if it is vertex-transitive and has uniform $d$-polytopes as tiles [5]. Recall that uniform polytopes may be defined inductively, declaring uniform polygons to be regular and uniform polytopes of rank 3 or higher to be vertex-transitive with uniform facets.

A 3-intransitive two-orbit tiling of 3-space has regular polyhedral tiles and is vertex-transitive, which means that it is a uniform tiling. Grünbaum [14] listed all 28 uniform tilings of 3 -space. Of these, only one is twoorbit: the tetrahedral-octahedral honeycomb, \#1 on Grünbaum's list, also called "alternated cubic," "Tetroctahedrille," or "octatetrahedral." This is 3 -intransitive. Coxeter describes it as the unique quasiregular honeycomb [8, p. 69] and assigns it the modified Schläfli symbol $\left\{3,{ }_{4}^{3}\right\}$ and an abbreviated symbol $h \delta_{4}$ [5, p. 402]. Being semiregular (with regular tiles and a vertex-transitive group), it also appears in Gosset's list [1] as the "simple tetroctahedric check." Monson and Schulte [23] describe this tiling at length. It has 6 octahedra and 8 tetrahedra meeting at each vertex; the vertex figure is a cuboctahedron. The corresponding "net," the 1-skeleton of the tiling, is named fcu by crystallographers in [10], where this tiling is conjectured to be the unique one with transitivity 1112 , i.e. whose symmetry group has one orbit on vertices, edges, and 2-faces, and two orbits on tiles.

A 2-intransitive tiling of 3-space has regular polygon 2-faces and is vertextransitive. Moreover, the facets are regular or 2-intransitive two-orbit, hence vertex-transitive. So such a tiling is uniform; but we already found the only two-orbit uniform tiling and this was 3-intransitive.

A 1-intransitive tiling of 3-space has two kinds of edge, which must alternate around a 2 -face, so each 2 -face has evenly many sides. The facets are regular or 1-intransitive two-orbit, and the only such polyhedron with even-sided 2 -faces is the cube. The only face-to-face tiling by cubes is the regular one. So no such tilings exist. 
A 0 -intransitive tiling of 3-space has two kinds of vertex, and every edge must be incident to one of each (by edge-transitivity), so each 2-face has evenly many sides. The facets are regular or 0-intransitive two-orbit; the only possibilities are the cube, the rhombic dodecahedron, or the rhombic triacontahedron. As we already mentioned, the only face-to-face tiling by cubes is regular. The rhombic triacontahedron has a dihedral angle of $4 \pi / 5$, so it is impossible to fit an integral number of them around an edge in 3space. However, the rhombic dodecahedron, with a dihedral angle of $2 \pi / 3$, does form a two-orbit tiling of 3 -space in a unique way. This tiling (called the rhombic dodecahedral honeycomb) is dual to the tetrahedral-octahedral honeycomb above. The corresponding net is named flu in [10], and described as the structure of fluorite $\left(\mathrm{CaF}_{2}\right.$.) It is conjectured there to be the unique tiling with transitivity 2111.

Suppose $\mathcal{T}$ is a fully transitive two-orbit tiling; then the facets are regular or two-orbit, and all of one type. Since $\mathcal{T}$ is vertex-transitive, if the facets were regular, $\mathcal{T}$ would be uniform, and we have already checked all the uniform tilings. Thus the facets must be two-orbit. Since $\mathcal{T}$ is 2 -facetransitive, every 2 -face is the same, which rules out the cuboctahedron or icosidodecahedron as facets. The remaining possibilities are the rhombic dodecahedron, which only appears in the 0-intransitive tiling already listed, and the rhombic triacontahedron, which as mentioned does not tile 3-space.

6.4. Higher dimensions. For a rank $(d+1)$ tiling $\mathcal{T}$ with $d \geq 4$, the facets and vertex figures are $d$-dimensional polytopes with at most two orbits. Since no two-orbit convex polytopes exist in $d \geq 4$ dimensions, the facets and vertex figures must, in fact, be regular; but then $\mathcal{T}$ itself is regular [8, p. 129].

\section{Conclusion}

The number of half-regular convex polytopes and tilings (to use Conway's pleasant term) is perhaps surprisingly small. Those which are also combinatorially two-orbit are simply the cuboctahedron and the icosidodecahedron, the only two quasiregular polyhedra, and their duals; the trihexagonal tiling, the only quasiregular plane tiling, and its dual; and the tetrahedraloctahedral honeycomb, the only quasiregular honeycomb, and its dual. It is notable, perhaps, that although duality is not generally well-defined for tilings, it always works well for two-orbit tilings which are combinatorially two-orbit, just as it always works well for regular tilings and uniform plane tilings. However, it does not generally work out for two-orbit tilings which are combinatorially regular!

The above seems suggestive that "quasiregular," which has previously had rather ad-hoc definitions, could be taken to mean "facet-intransitive two-orbit." Coxeter [8, p. 18] defines a "quasi-regular polyhedron" as "having regular faces, while its vertex figures, though not regular, are cyclic and equi-angular (i.e., inscriptible in circles and alternate-sided)." The definition 
of a quasiregular plane tiling does not seem to be clearly stated, but the implication (in $[8, \S 4.2]$ ) is that a quasiregular plane tiling is one formed, as the quasiregular polyhedra can be, by truncating the vertices of a regular tiling to the midpoints of the edges. In $[8, \S 4.7]$, a tiling of 3 -space (or honeycomb) "is said to be quasi-regular if its cells are regular while its vertex figures are quasi-regular." This suggests the beginning of an inductive definition for "quasiregular" in higher dimensions, which would perhaps agree with ours: Facet-intransitive two-orbit polytopes have regular facets and the vertex figures are again facet-intransitive and two-orbit. It would be good to establish that having regular facets and facet-intransitive two-orbit vertex figures implies that the polytope is two-orbit. This is vacuously true for convex polytopes, since Blind [2] classified all regular-faced $d$-polytopes with $d \geq 4$, and none have two-orbit vertex figures. However, the corresponding result for abstract polytopes would clarify the agreement of the definitions.

The word "quasiregular" is also applied to some star polytopes, such as the dodecadodecahedron $\left\{\begin{array}{c}5 \\ 5 / 2\end{array}\right\}$ and the great icosidodecahedron $\left\{\begin{array}{c}3 \\ 5 / 2\end{array}\right\}$ in [8, pp. 100-101]; three ditrigonal forms: the ditrigonal dodecadodecahedron, small ditrigonal icosidodecahedron, and great ditrigonal icosidodecahedron (also called "triambic" instead of "ditrigonal"); and nine hemihedra: the tetrahemihexahedron, octahemioctahedron, cubohemioctahedron, small icosihemidodecahedron, small dodecahemidodecahedron, great dodecahemicosahedron, small dodecahemicosahedron, great dodecahemidodecahedron, and great icosihemidodecahedron (using names from [27]). All of these are two-orbit facet-intransitive. It would be good to establish that these are the only two-orbit facet-intransitive star polytopes.

Remaining questions include the classification of two-orbit tilings of hyperbolic space, two-orbit star polytopes, and other non-convex two-orbit polytopes in Euclidean space. The general abstract two-orbit polyhedra have been addressed in [18], with extension to higher dimensions in preparation [19]. An overview is in [17, §1.3]. The important special case of chiral polytopes have been studied extensively but many open questions remain; a recent survey is [25].

It also remains to classify convex polytopes of three or more orbits. Results in this direction, mostly for abstract polytopes, are found in [9], [17], and [24].

\section{Acknowledgments}

The author would like to thank his adviser, Egon Schulte, for his guidance and assistance, and suggesting the original problem, and Peter McMullen for suggesting improvements. 


\section{REFERENCES}

[1] G. Blind and R. Blind. "The semiregular polytopes". In: Commentarii Mathematici Helvetici 66.1 (Dec. 1991), pp. 150-154. ISSN: 0010-2571. DOI: $10.1007 / \mathrm{BF} 02566640$.

[2] R. Blind. "Konvexe Polytope mit regulären Facetten im $\mathbb{R}^{n}(n \geq 4)$ ". In: Contributions to Geometry: Proceedings of the geometry symposium held in Siegen, 1978. Ed. by J. Tölke and J. Wills. 1979, pp. 248-254.

[3] M. Brückner. Über die gleicheckig-gleichflächigen, diskontinuierlichen und nichtkonvexen Polyeder. Die Kaiserl. Leop.-Carol. Deutsche Akademie der Naturforscher, 1906.

[4] J. H. Conway, H. Burgiel, and C. Goodman-Strauss. The Symmetries of Things. AK Peters, 2008.

[5] H. S. M. Coxeter. "Regular and semi-regular polytopes. I". In: Mathematische Zeitschrift 46.1 (1940), pp. 380-407.

[6] H. S. M. Coxeter. "Regular and semi-regular polytopes. II". In: Mathematische Zeitschrift 188.4 (1985), pp. 559-591.

[7] H. S. M. Coxeter. "Regular and semi-regular polytopes. III". In: Mathematische Zeitschrift 200.1 (1988), pp. 3-45.

[8] H. S. M. Coxeter. Regular Polytopes. Third edition. Dover Publications, Inc., 1973. ISBN: 0-486-61480-8.

[9] G. Cunningham. "Orbit graphs and face-transitivity of k-orbit polytopes". In: arXiv preprint arXiv:1212.2937 (2012).

[10] O. Delgado Friedrichs, M. O'Keeffe, and O. M. Yaghi. "Three-periodic nets and tilings: regular and quasiregular nets". In: Acta Crystallographica Section A: Foundations of Crystallography 59.1 (2002), pp. 22 27.

[11] T. Gosset. "On the regular and semi-regular figures in space of $\mathrm{n}$ dimensions". In: Messenger of Mathematics 29 (1900), pp. 43-48.

[12] J. E. Graver and M. E. Watkins. Locally finite, planar, edge-transitive graphs. Vol. 601. American Mathematical Soc., 1997.

[13] B. Grünbaum. Convex polytopes. Wiley, 1967.

[14] B. Grünbaum. "Uniform tilings of 3-space". In: Geombinatorics 4.2 (1994), pp. 49-56.

[15] B. Grünbaum and G. Shephard. "Edge-transitive planar graphs". In: Journal of Graph Theory 11.2 (1987), pp. 141-155.

[16] B. Grünbaum and G. C. Shephard. Tilings and patterns. W.H. Freeman \& Company, 1986.

[17] I. Helfand. "Constructions of k-orbit Abstract Polytopes". PhD thesis. Northeastern University Boston, Apr. 2013.

[18] I. Hubard. "Two-orbit polyhedra from groups". In: European Journal of Combinatorics 31.3 (2010), pp. 943-960.

[19] I. Hubard and E. Schulte. "Two-orbit polytopes". In preparation.

[20] N. W. Johnson. Uniform Polytopes. Cambridge University Press, to appear. 
[21] P. McMullen. "On the combinatorial structure of convex polytopes". $\mathrm{PhD}$ thesis. University of Birmingham, June 1968.

[22] P. McMullen and E. Schulte. Abstract regular polytopes. Cambridge University Press, 2002.

[23] B. Monson and E. Schulte. "Semiregular polytopes and amalgamated C-groups". In: Advances in Mathematics 229.5 (2012), pp. 2767-2791.

[24] A. Orbanić, D. Pellicer, and A. I. Weiss. "Map operations and k-orbit maps". In: Journal of Combinatorial Theory, Series A 117.4 (2010), pp. 411-429.

[25] D. Pellicer. "Developments and open problems on chiral polytopes". In: Ars Mathematica Contemporanea 5.2 (2012).

[26] E. Schulte and A. I. Weiss. "Chiral polytopes". In: Applied Geometry and Discrete Mathematics ("The Victor Klee Festschrift"), DIMACS Series in Discrete Mathematics and Theoretical Computer Science 4 (1991), pp. 493-516.

[27] M. J. Wenninger. Polyhedron models. Cambridge University Press, 1974.

Department of Mathematics, 567 Lake Hall, Northeastern University, Boston, MA 02115

E-mail address: matteo.n@husky.neu.edu 